\title{
La grossesse chez les hommes transgenres
}

\author{
Kimberley G.S. Thornton MD BKin, Fiona Mattatall MD MSc
}

Citation : CMAJ 2021 August 23;193:E1303. doi : 10.1503/cmaj.210013-f

Voir la version anglaise de l'article ici : www.cmaj.ca/lookup/doi/10.1503/cmaj.210013

\section{Il faudrait systématiquement aborder la question de la fertilité avec les hommes transgenres}

Les professionnels de la santé devraient discuter régulièrement de fertilité avec les hommes transgenres, surtout ceux qui ont un utérus, sans faire de suppositions sur leurs activités sexuelles ou leurs objectifs génésiques ${ }^{1}$. Ils devraient parler aux patients qui souhaitent porter un enfant du processus médical et psychosocial auquel ils peuvent s'attendre, depuis la préconception jusqu'à la période postnatale ${ }^{2,3}$.

2

\section{La testostérone n'est pas un moyen de contraception, même chez \\ les patients sans menstruations}

Les hommes transgenres qui ont un utérus risquent de vivre une grossesse non planifiée. La documentation sur le sujet, quoique limitée, semble montrer que cette population présente un taux de grossesses non planifiées aussi élevé que celui des femmes cisgenres, et utilise dans des proportions semblables toutes les formes de contraception, des méthodes barrières aux contraceptifs hormonaux ${ }^{1}$. Il est possible de prendre des contraceptifs oraux et de suivre une hormonothérapie androgénique en même temps.

\section{3}

\section{Les consultations préconceptionnelles des hommes transgenres \\ doivent inclure une discussion sur la poursuite de la prise de} testostérone

La testostérone entraîne des effets tératogènes. Elle peut causer des anomalies du développement urogénital chez le fotus féminin. Il vaut donc mieux ne pas en prendre avant la conception et pendant la grossesse $\mathrm{e}^{1,4}$.

\section{On ne sait pas si le risque de complications périnatales est accru chez les hommes transgenres}

II n'y a pas de données de grande qualité sur l'incidence des complications de la grossesse (diabète gestationnel, hypertension, etc.) chez les hommes transgenres'. L'accouchement vaginal est possible; cela dit, le professionnel doit présenter toutes les options au patient pour optimiser son expérience du libre choix et de l'accouchement ${ }^{2,3,5}$. L'allaitement peut être envisagé après la naissance, si le patient le désire ${ }^{2,3}$.

5 L

Le soutien psychosocial est un volet essentiel du suivi de grossesse pour les hommes transgenres

Il existe peu de ressources sur la grossesse spécialement conçues pour les hommes transgenres, mais les besoins n'en sont pas moins grands ${ }^{2}$. Ces patients craignent souvent d'être jugés par leur communauté, la population en général et les fournisseurs de soins. Pour leur prodiguer des soins inclusifs et adaptés, il faut faire attention au contexte ainsi qu'aux pronoms et aux mots employés (par exemple dire « poitrine » plutôt que « seins ») $)^{2,3,5}$.

\section{Références}

1. Light A, Wang L-F, Zeymo A, et al. Family planning and contraception use in transgender men. Contraception 2018;98:266-9.

2. Light AD, Obedin-Maliver J, Sevelius JM, et al. Transgender men who experienced pregnancy after female-to-male gender transitioning. Obstet Gynecol 2014;124:1120-7.

3. Hoffkling A, Obedin-Maliver J, Sevelius J. From erasure to opportunity: a qualitative study of the experiences of transgemder men around pregnancy and recommendations for providers. BMC Pregnancy Childbirth 2017;17(Suppl 2):332.

4. Krempasky C, Harris M, Abern L, et al. Contraception across the transmasculine spectrum. Am J Obstet Gynecol 2020;222:134-43.

5. Brandt JS, Patel AJ, Marshall I, et al. Transgender men, pregnancy, and the "new" advanced paternal age: a review of the literature. Maturitas 2019;128:17-21.

Intérêts concurrents : Fiona Mattatall copréside le groupe de travail sur le transgenrisme de la Société des obstétriciens et gynécologues du Canada. Aucun autre intérêt concurrent n'a été déclaré.

Cet article a été révisé par des pairs.

Affiliations : Département d'obstétrique et de gynécologie, Faculté de médecine Cumming, Université de Calgary, Calgary, Alb.

Propriété intellectuelle du contenu : Il s'agit d'un article en libre accès distribué conformément aux modalités de la licence Creative Commons Attribution (CC BY-NC-ND 4.0), qui permet l'utilisation, la diffusion et la reproduction dans tout médium à la condition que la publication originale soit adéquatement citée, que l'utilisation se fasse à des fins non commerciales (c.-à-d., recherche ou éducation) et qu'aucune modification ni adaptation n'y soit apportée. Voir : https://creativecommons.org/ licenses/by-nc-nd/4.0/deed.fr.

Correspondance : Kimberley Thornton, kimberley.thornton@albertahealthservices.ca

Le JAMC vous invite à soumettre vos textes pour la rubrique « Cinq choses à savoir ... » en ligne à http://mc.manuscriptcentral.com/cmaj. 